\title{
Motor unit size in muscular dystrophy, a macro EMG and scanning EMG study
}

\author{
PER HILTON-BROWN, ERIK STÅLBERG \\ From the Department of Clinical Neurophysiology, University Hospital, Uppsala, Sweden
}

SUMMARY Patients with muscular dystrophy were investigated with Macro EMG to study activity from whole individual motor units, and with Scanning EMG to study the distribution of activity within the motor unit. Macro motor unit potentials were normal or only slightly reduced in amplitude. In Scanning EMG the units had unchanged mean length compared with normal, but an uneven distribution of the activity. This was also seen in severely weak muscles. The findings are interpreted to be the result of degenerative and regenerative processes, giving rise to remodelling of the motor unit.

The local fibre density (FD) measured with single fibre electromyography (SFEMG) is increased in muscular dystrophies. ${ }^{1-3}$ An increased FD is commonly seen in neurogenic disorders where it is interpreted as a sign of reinnervation, giving dense motor units with increased number of fibres. ${ }^{4}$ In these cases the concentric needle EMG (CNEMG) also indicates an increase in number of muscle fibres in the motor unit within the pick-up area of the electrode. In muscular dystrophies, however, CNEMG does not indicate dense motor units. Instead the typical findings of reduced duration and amplitude of the motor unit potentials are considered to reflect smaller than normal motor units. ${ }^{5}$ Thus in muscular dystrophies the two techniques give apparently contradictory findings. This may be due to the difference in pick up area of the two electrodes, although the concentric electrode is also relatively selective, recording from only a small part of the entire motor unit. ${ }^{6}$ In order to estimate whether the dystrophic motor unit is small, as suggested by CNEMG or large as might be concluded from the SFEMG recordings, information about the whole motor unit should be of value. Techniques have recently been developed that provide such information. With Macro EMG, a less selective recording of the electrical activity from the whole motor unit is obtained. ${ }^{7}$ In this method the activity in a voluntarily activated motor unit is recorded from the large bare terminal portion of an intramuscular electrode. With

Address for reprint requests: Dr Per Hilton-Brown, Dept of Clin Neurophysiology, University Hospital, S-75185 Uppsala, Sweden.

Received 22 April 1983. Accepted 14 May 1983
Scanning EMG the spatial and temporal distribution of the motor unit can be studied. ${ }^{8}$ The aim of this study was to investigate fibre density and the total size of motor units in muscular dystrophy by means of Macro EMG and Scanning EMG.

\section{Patients}

Seven patients with limb girdle (LG) and 11 with facioscapulo-humeral (FSH) muscular dystrophy were studied. The patients had participated in our earlier studies. For diagnoses and clinical evaluation see reference 3 . The patients were graded for general disability (grade $0=$ preclinical, $10=$ confined to bed), for rate of progression during the last two years, and for strength (grade $0=$ paralysis, $7=$ full strength) and atrophy in brachial biceps and tibial anterior muscles (see fig 2, ref 3 where the same patient numbers are used as here).

\section{Methods}

\section{Macro EMG}

The Macro EMG electrode is a modified SFEMG electrode which is insulated to within $15 \mathrm{~mm}$ of the tip. A single fibre electrode surface is exposed in a side-port in the middle of the bare part. A two channel recording is made. On one channel the Macro EMG is recorded between the bare cannula and a remotely placed subcutaneous reference electrode. Filter settings of the amplifier are $8 \mathrm{~Hz}$ and $8 \mathrm{KHz}$. On the second channel a SFEMG recording is made from the SFEMG surface with the cannula acting as reference. The electrode is introduced perpendicularly to the expected direction of the muscle fibres, $20-30 \mathrm{~mm}$ away from the supposed end-plate area. The whole uninsulated part of the electrode must be within the muscle. The needle is positioned to obtain steady single fibre activity. The single fibre action potential is used to trigger the aver- 
ager and the oscilloscope sweep. The number of spike components in the triggering SFEMG recording is used to calculate FD. In order to include also activity preceding the triggering moment, the Macro signal is delayed $40 \mathrm{~ms}$ and fed to a computer, in which $80 \mathrm{~ms}$ ( $40 \mathrm{~ms}$ before and after the triggering) of the sweep is averaged in two alternating buffers. The averaging is accepted when the point-to-point difference in the two buffers is less than a preset value which is typically reached after 20-200 epochs of averaging. The obtained signal is called the Macro motor unit potential, Macro MUP. In the analysis of the Macro MUP the initial and terminal $10 \mathrm{~ms}$ are used to define the baseline. The peak to peak amplitude and the area under the signal during the remaining $60 \mathrm{~ms}$ is calculated.

Activity from motor units that may have a tendency to discharge synchronously with the studied motor unit may disturb the recording. In contrast to the rigid time locking between components in the same motor unit (jitter less than $0.1 \mathrm{~ms}$ ) there is a large variation in the locking between action potentials from different motor units also when they tend to fire together. The term semisynchronous is used to denote this phenomenon. As a control of the semisynchronous activity the following procedure is used. In a separate memory the rectified Macro signal is averaged. When the data collection is finished the averaged original signal is rectified and subtracted from the resulting average of the rectified signal. A deflection in the remaining signal will indicate semisynchronous activity and the off-set the amount of background activity. Recordings indicating semisynchronous activity were not accepted. For further technical details of Macro EMG see separate report.'

With varying transverse recording positions at the same end-plate distance the amplitude of the Macro MUP may differ but the shape characteristics are usually preserved in normal muscles. Thus it can usually be recognised if two recordings are made from the same motor unit. In a complete study at least 20 different potentials are obtained.

Macro MUP amplitude and area vary with age and show a skew distribution in the normal material. To obtain comparable values from patients of different ages relative amplitude and area values for individual Macro MUPs were calculated and used in statistical analysis. (= measured value/normal median for age). To obtain a normal distribution of data for statistical calculations the logarithm of the relative values were used.

A study was considered abnormal if either more than one Macro Motor Unit Potential (MUP) was below or above the normal limits, or if the median value in the study was outside its normal limits. ${ }^{79}$

A computer pattern recognition programme based on the same normal material as used here, has been presented. ${ }^{10}$ Its primary aim is to detect Macro MUPs with abnormal characteristics in addition to amplitude and area. The programme is at present used for analysis of Macro MUPs with amplitudes exceeding $150 \mu \mathrm{V}$, in the brachial biceps muscle in subjects of more than 40 years of age. Potentials recorded in dystrophic patients were analysed with this programme to test whether Macro MUPs with normal amplitudes were abnormal.

Macro EMG studies were performed in the brachial biceps and tibial anterior muscles. These muscles were chosen because they represent proximal and distal muscles, they have comparatively parallel fibre arrangements and their bulk is well suited for Macro EMG.

The fibre density (FD) was calculated from the number of components in the triggering SFEMG recording according to standard criteria. ${ }^{11}$ The fibre density is expressed as relative fibre density RFD = measured FD/mean FD for age.

\section{Scanning EMG}

So called Scanning EMG was performed according to an earlier described method. ${ }^{8} \mathrm{~A}$ single fibre electrode was positioned in the voluntarily activated muscle and activity from a single muscle fibre was used as trigger signal. A concentric EMG needle was introduced perpendicularly to the fibre direction $1-3 \mathrm{~cm}$ from the single fibre needle and positioned so that maximal motor unit activity synchronous to the triggering fibre (that is, belonging to the same motor unit) was obtained. The concentric electrode was then pushed slightly deeper into the muscle, until the motor unit no longer was recorded. A step-motor with 50 micron steps was connected. The signals from the concentric EMG needle were delayed, A/D-converted and stored in a computer. The step motor was controlled by the computer, and it pulled the needle one step per sweep. The consecutive signals were plotted. The motor unit activity and its distribution along the retraction path of the needle in the muscle was thus depicted. A profile of the peak-to-peak amplitude and area of the unit along the retraction path was plotted by the computer. The length through which unit activity with an amplitude exceeding $50 \mu \mathrm{V}$ was measured. The number of subdivisions in this length, separated by amplitudes less than $50 \mu \mathrm{V}$, "silent areas", were counted. With the cannula inside the studied motor unit and with the tip outside a signal with reversed polarity was seen compared with that obtained from the tip in an active area. This signal, "the trough" has a constant amplitude during the electrode withdrawal until the tip is close to active fibres. The trough was not included in the measurements of the signal.

\section{Results}

Fibre density in the brachial biceps and the tibial anterior muscle were significantly increased compared with normal (table 1).

The Macro MUP amplitude was abnormally low in 15 , normal in 16 and abnormally high in four of the 35 muscles studied (figs 1, 2, 3 and table 1). As seen in fig 2 most Macro MUPs had amplitudes within the normal range. The pooled Macro MUPs from all the patients showed a slight but significant decrease in amplitudes compared with normals. This was slightly more pronounced in the FSH group than in LG, and slightly more so in tibialis anterior than in biceps muscles $(p<0.01)$. The mean Macro MUP area was not significantly different from normal except in tibialis anterior in the FSH group where it was decreased.

The recordings were usually easily obtained even 
Table 1 Summary of Macro EMG data

\begin{tabular}{|c|c|c|c|c|c|c|c|c|c|c|}
\hline No & \multirow[t]{2}{*}{$R F D$} & \multirow{2}{*}{$\begin{array}{l}\text { FD no of } \\
\text { pathol } \\
\text { invest }\end{array}$} & \multirow{2}{*}{$\begin{array}{l}\text { MUP } \\
\text { median } \\
\text { ampl } \\
(\mu V)\end{array}$} & \multirow{2}{*}{$\begin{array}{l}\text { Change } \\
\text { from } \\
\text { normal } \\
\text { in mean } \\
\text { Macro } \\
\text { MUP } \\
\text { ampl } \\
(\%)\end{array}$} & \multirow{2}{*}{$\begin{array}{l}\text { Change } \\
\text { from } \\
\text { normal } \\
\text { in mean } \\
\text { Macro } \\
\text { MUP } \\
\text { area } \\
(\%)\end{array}$} & \multicolumn{3}{|c|}{ Macro MUP area-ampl } & \multirow{2}{*}{$\begin{array}{l}\text { No of } \\
\text { Macro } \\
\text { invest } \\
\text { pathol } \\
\text { decreased }\end{array}$} & \multirow{2}{*}{$\begin{array}{l}\text { No of } \\
\text { Macro } \\
\text { invest } \\
\text { pathol } \\
\text { increased }\end{array}$} \\
\hline & & & & & & Ratio & $\begin{array}{l}\text { Correlation } \\
(r)\end{array}$ & $\begin{array}{l}\text { Ratio } \\
\text { in MUPs } \\
\text { with } \\
\text { ampl } \\
>150 \mu V\end{array}$ & & \\
\hline \multicolumn{11}{|l|}{ Biceps brachii. } \\
\hline $\begin{array}{lr}\text { Normal } & 35 \\
\text { LG } & 7 \\
\text { FSH } & 10 \\
\text { LG + FSH } & 17\end{array}$ & $\begin{array}{l}1.000 \\
1.540 \dagger \\
1.265 \dagger \\
1.378^{*}\end{array}$ & $\begin{array}{r}0 \\
7 \\
6 \\
13\end{array}$ & $\begin{array}{r}102 \\
94 \\
86 \\
89\end{array}$ & $\begin{array}{c}0 \\
-14 \cdot 4^{*} \\
-18 \cdot 1^{*} \\
-16 \cdot 6^{*}\end{array}$ & $\begin{array}{l}0 \\
+\quad 5.6 \S \\
+2.9 \S \\
+4.0 \$\end{array}$ & $\begin{array}{l}3 \cdot 4 \\
4 \cdot 2 \\
3 \cdot 9 \\
4 \cdot 0\end{array}$ & $\begin{array}{l}0.84 \\
0.81 \\
0.78 \\
0.79\end{array}$ & $\begin{array}{l}3 \cdot 0 \\
2 \cdot 4 \\
2 \cdot 5 \\
2 \cdot 5\end{array}$ & $\begin{array}{l}0 \\
3 \\
4 \\
7\end{array}$ & $\begin{array}{l}0 \\
1 \\
1 \\
2\end{array}$ \\
\hline \begin{tabular}{lc}
\multicolumn{2}{l}{ Tibialis } \\
Normal & 35 \\
LG & 7 \\
FS & 11 \\
FSH & 18
\end{tabular} & $\begin{array}{l}1.000 \\
1.441 \dagger \\
1.246 \dagger \\
1.322^{*}\end{array}$ & $\begin{array}{r}0 \\
5 \\
5 \\
10\end{array}$ & $\begin{array}{l}172 \\
155 \\
118 \\
132\end{array}$ & $\begin{array}{c}0 \\
-10 \cdot 1 \dagger \\
-31 \cdot 8^{*} \\
-23 \cdot 0^{*}\end{array}$ & $\begin{array}{c}0 \\
+16 \cdot 9 \S \\
-\quad 8 \cdot 3^{*} \\
+\quad 2 \cdot 0 \ddagger\end{array}$ & $\begin{array}{l}3 \cdot 8 \\
4 \cdot 6 \\
5 \cdot 4 \\
5 \cdot 1\end{array}$ & $\begin{array}{l}0.89 \\
0.81 \\
0.83 \\
0.83\end{array}$ & $\begin{array}{l}3 \cdot 6 \\
2 \cdot 8 \\
3 \cdot 4 \\
3 \cdot 2\end{array}$ & $\begin{array}{l}0 \\
2 \\
6 \\
8\end{array}$ & $\begin{array}{l}0 \\
0 \\
2 \\
2\end{array}$ \\
\hline
\end{tabular}

In $t$ test when compared to normal, ${ }^{*} \mathrm{p}<0.001,+\mathrm{p}<0.01, \neq \mathrm{p}<0.05$, §not significant

A

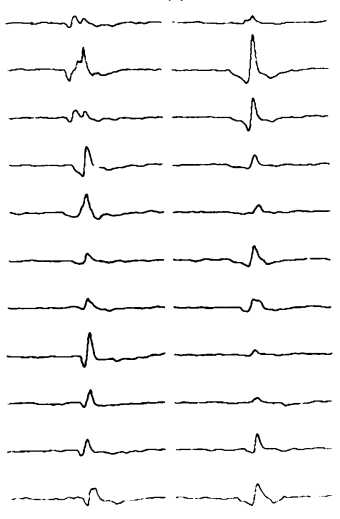

B

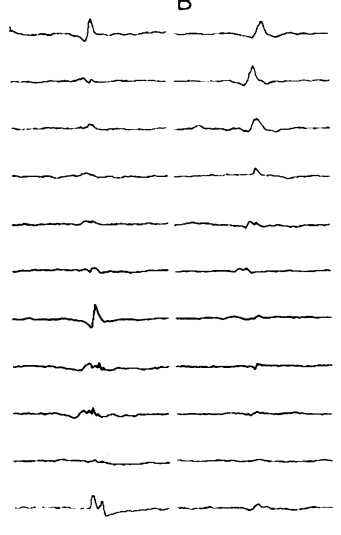

C

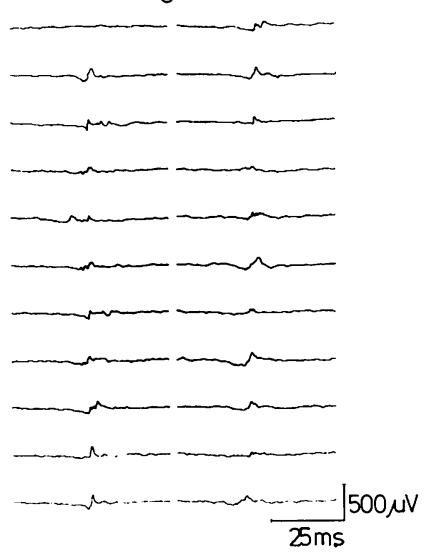

Fig 122 Macro MUPs recorded from different positions in biceps brachii in a normal control $(A)$, and in patients with $L G(B)$ and $F S H(C)$ dystrophy.

in severely weakened muscles. The dystrophic patients, even at slight activation often recruit several motor units which fire at high innervation rates. Consequently there was often more "background activity" with an excess of high frequency components than is usually seen in normal subjects. To minimise the background activity, most recordings, as in the normal material ${ }^{7}{ }^{9}$ were made at low activity. With increasing activity larger MUPs seemed to be recruited, but this was not systematically studied. Some potentials were small, corresponding to double the noise level. Records, with no detectable Macro MUPs were only seen when a fibrillation potential was used as trigger signal. (These record- ings were not included in the statistics). There was a tendency for the units to contain more peaks than in normals.

In two studies a separate single fibre electrode was used to obtain a constant triggering signal. The Macro EMG electrode was positioned adjacent in the direction of the long axis of the muscles but with a lateral displacement from the SFEMG needle of 0 , $5,10,15$ and $20 \mathrm{~mm}$ in both directions. 10 MUPs were studied in this way. There was a uniform increase and decline of Macro MUP amplitude and area with the lateral displacement but some units varied more in shape than was seen in normals (fig 4). 


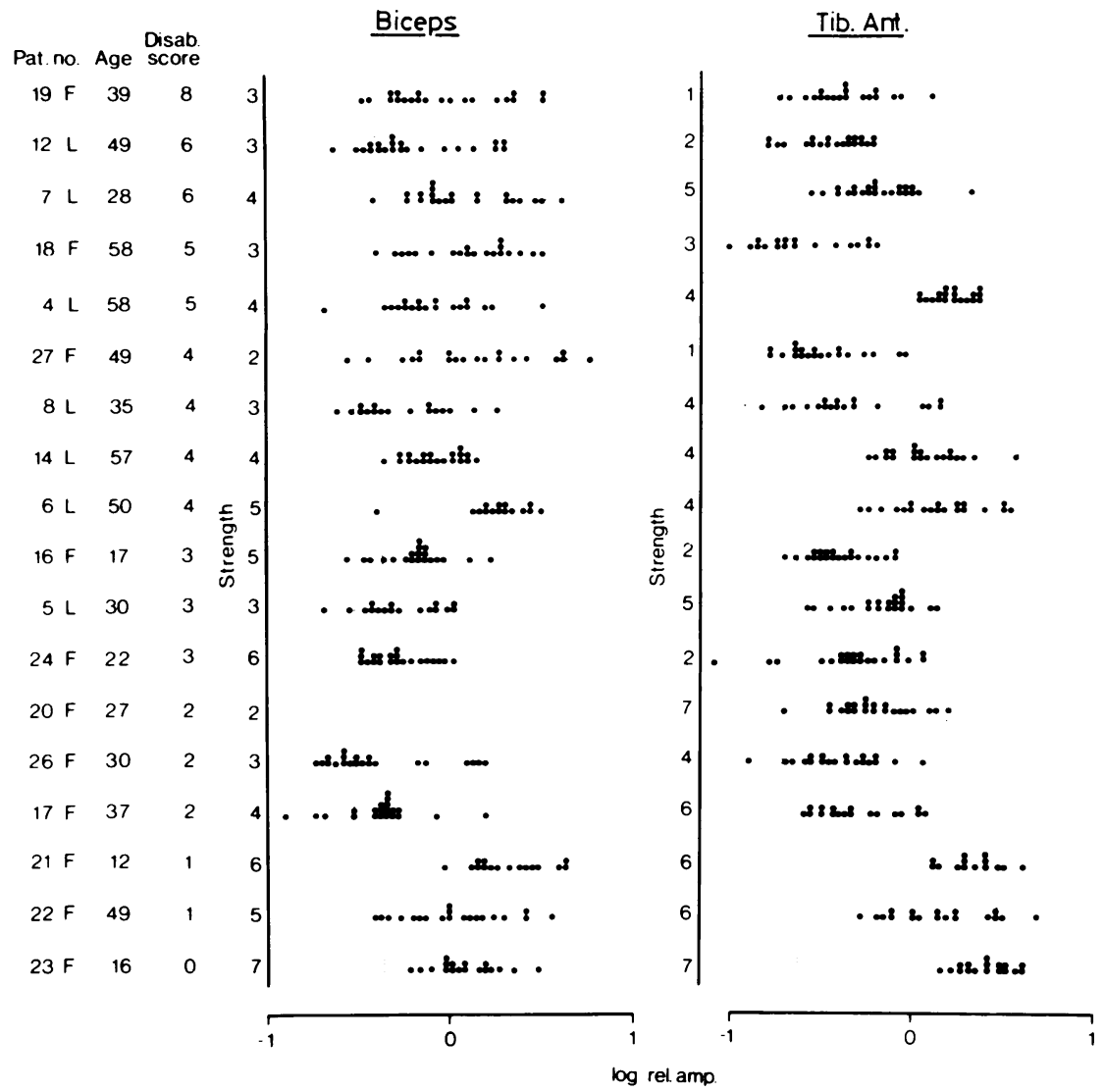

Fig 2 Amplitudes of individual Macro MUPs for the $L G(L)$ and FSH $(F)$ patients in biceps and tibialis anterior muscles. Values are given as the logarithm of the ratio of the recorded amplitude/normal median for age $\cdots$ indicates upper and lower normal limits for age, for individual Macro MUPs. The material is sorted with decreasing general disability score. Patient numbers are the same as in ref 3 . Some patients did not participate in both studies, therefore some numbers are missing.

In two patients the effect of interference from other units on the Macro MUP was studied. The same Macro MUPs were recorded during minimal contraction when the Macro unit was visible without averaging and during more forceful contraction, when it was hidden in the background activity. The units could increase in amplitude and area, due mainly to an increased late positive phase. There was usually a computer indication of semisynchronous activity, which resulted in rejection of the recording. When there was no such indication, the Macro MUPs showed little or no increase (up to $15 \%$ amplitude increase), even when the background activity was clearly higher than that which occurred in the main investigation (fig 5).
The patients were also instructed to produce a slight voluntary tremor in the studied muscle. This was detected by the computer before any increase in the MUP was noted.

The computer pattern recognition programme was tried on the MUPs from the six patients who fell within the required age limit. Of the 52 MUPs with amplitudes above $150 \mu \mathrm{V}, 46 \%$ were classified as abnormal. Of the total 136 recorded MUPs in these patients $2.2 \%$ were outside the amplitude limits.

In biceps RFD and mean of the relative Macro MUP amplitude showed a positive correlation $\mathrm{r}=$ $0.71(p<0.004)$. In tibialis anterior there was a positive correlation between strength and mean of the relative Macro amplitude $r=0.55(\mathrm{p}<0.01)$. 


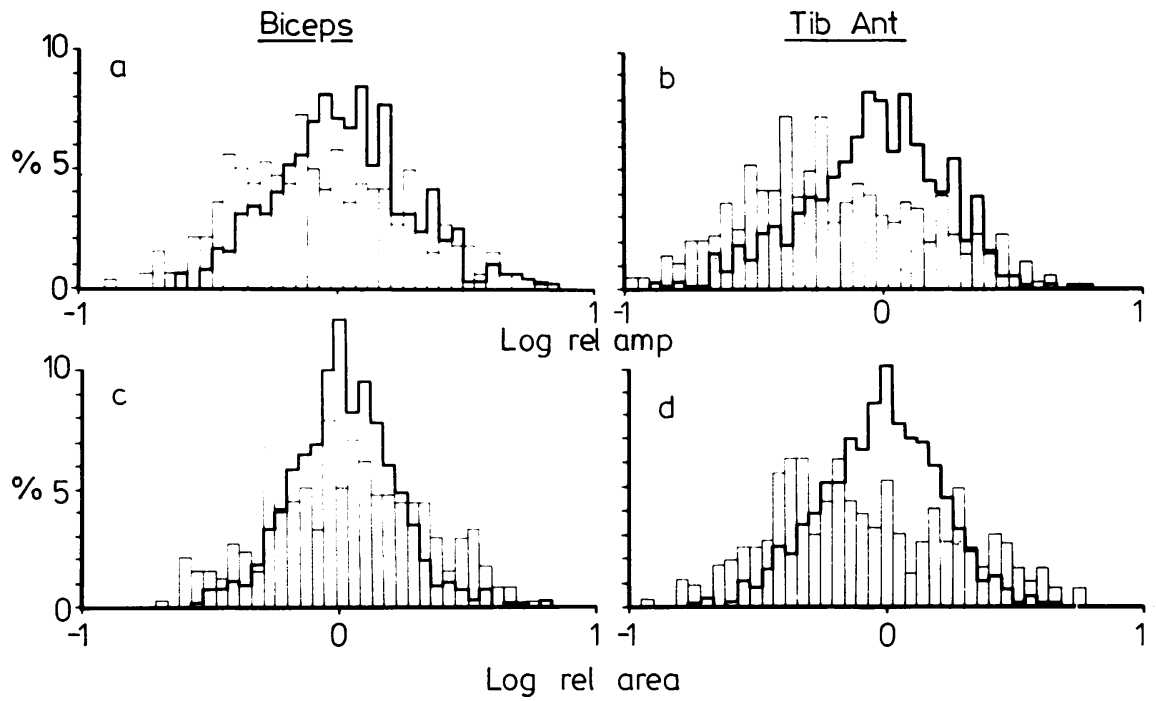

Fig 3 Histogram of MUP amplitude ( $A$ and $B$ ) and area ( $C$ and $D)$. The values are given as logarithm of the ratio of recorded value, /normal median for age. Normal material indicated with full lines, pooled dystrophy material as columns.

Biceps
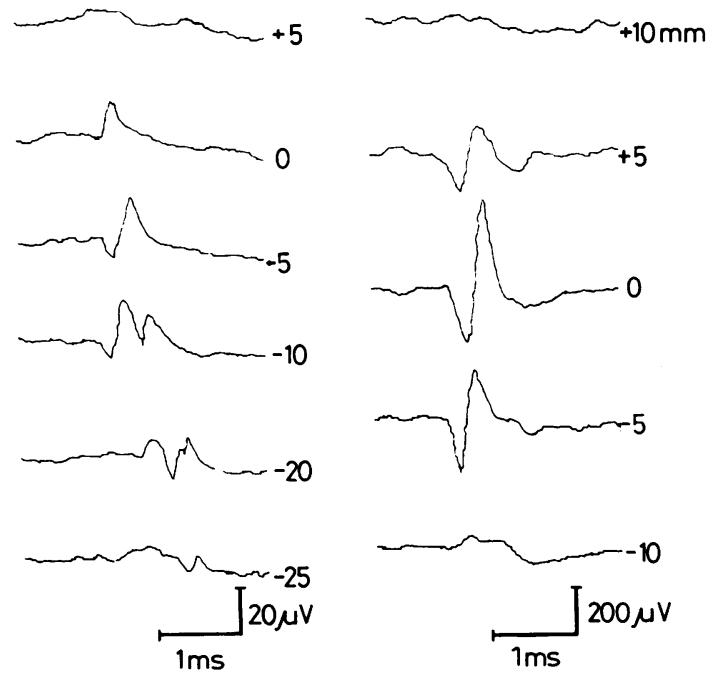

There was no other significant correlation between Macro amplitude or RFD and the clinical parameters, of age, duration of the disease, serum creatineinase, grading for total disability, progression, strength or atrophy.

Scanning EMG was performed in five patients with LG and in two with FSH dystrophy. The investiga-
Fig 4 Repeated Macro recordings in a FSH dystrophy from one motor unit at varying lateral displacement. In this biceps brachii recording there is a greater variation in the unit configuration and latency than is seen in normals, whereas the tibialis anterior recording has a normal variation. tion was performed in the tibial anterior and brachial biceps and tibiali anterior muscles in the were moderately to severely weak. Scanning EMG was also performed in four healthy controls. For results see table 2, figs 6 and 7 . The territory size has been reported in a separate study. ${ }^{3}$ There was no significant difference between dystrophic and nor- 

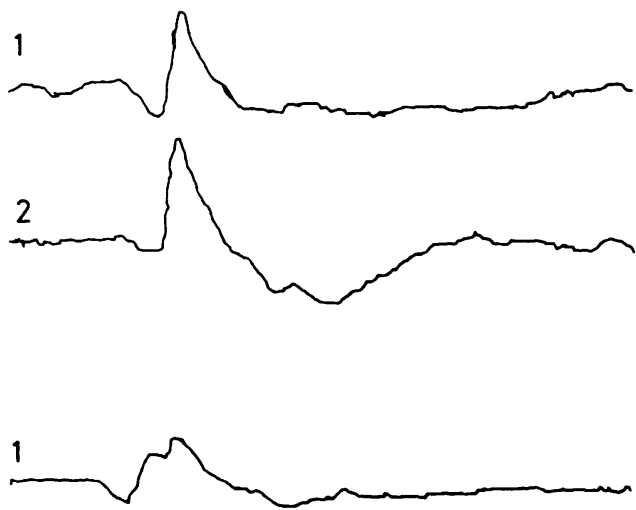

B

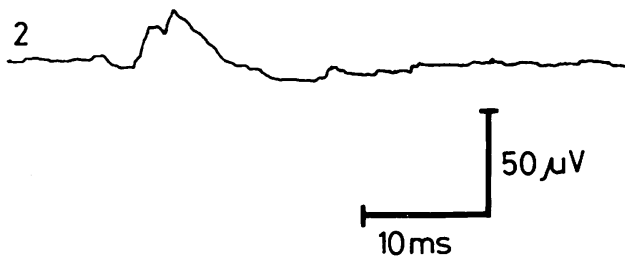

A

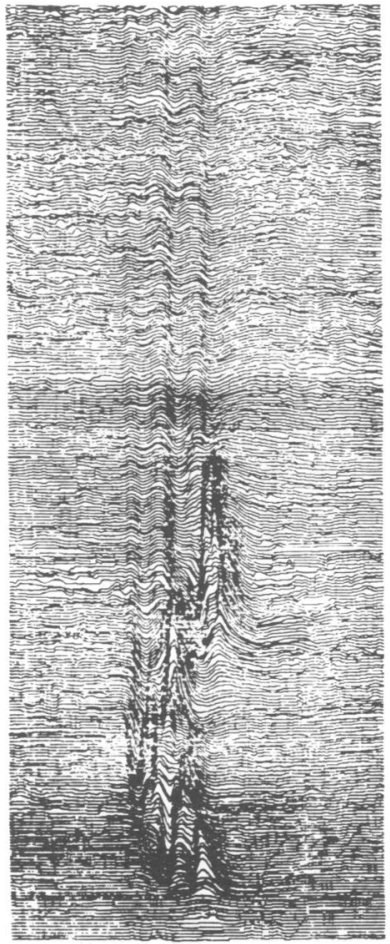

2

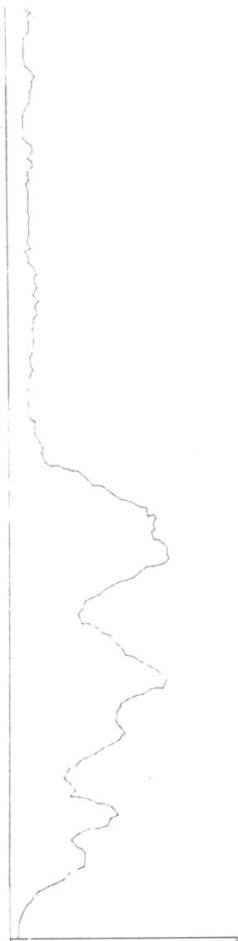

Fig 5 Macro MUPs recorded (1) at minimal activation (2) at more forceful activation A: Increase in amplitude. Note increase in late positive peak. There was a clear indication by the computer of semisynchronous activity in this recording. B: No change in amplitude.
B

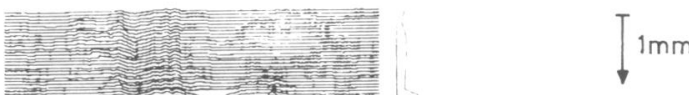

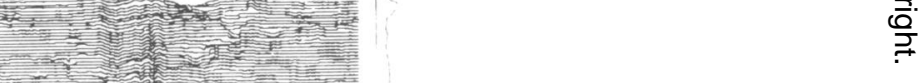

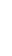
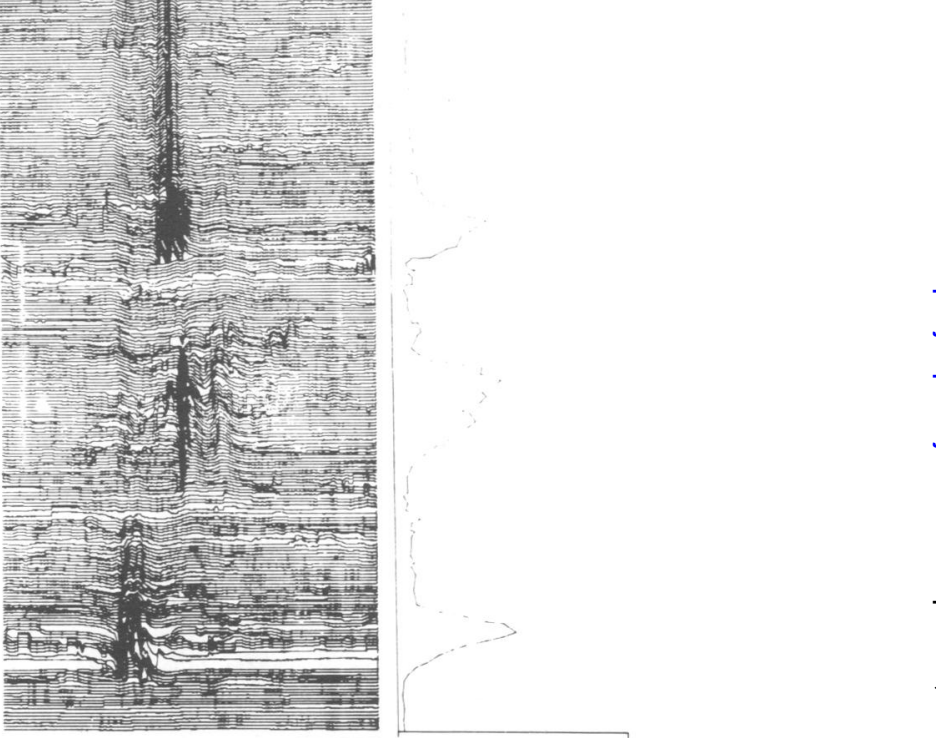

$5 \mathrm{~ms} 0$

$1 \mathrm{mV}$

Fig 6 Scanning EMG in tibialis anterior. A. normal muscle, B. FSH dystrophy. The peak-to-peak amplitude in each sweep is measured and indicated to the right of the scans $(A 2, B 2)$. Note that the total size of the territory is in this example slightly longer in the dystrophic muscle, where the unit activity is divided by silent areas. 

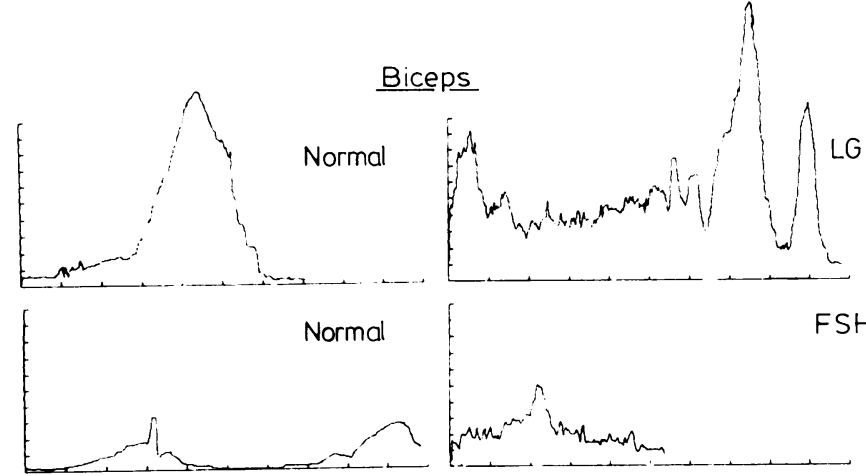

Tibialis anterior

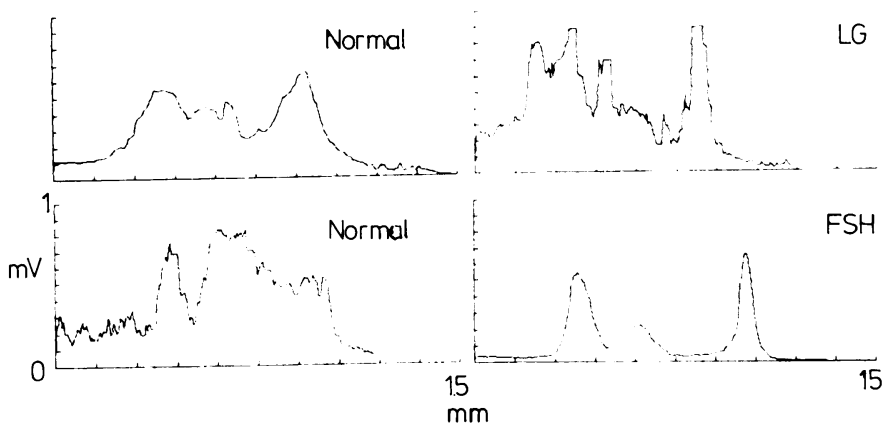

Fig 7 Examples of amplitude profiles of Scanning EMG in biceps brachii and tibialis anterior muscles from normals and from patients with $L G$ or FSH dystrophies.
Table 2 Summary of Scanning EMG data

\begin{tabular}{llll}
\hline & $\begin{array}{l}\text { No of } \\
\text { scans }\end{array}$ & $\begin{array}{l}\text { Mean total } \\
\text { length }\end{array}$ & $\begin{array}{l}\text { No of silent } \\
\text { areas mean/scan }\end{array}$ \\
\hline Biceps brachii & & & \\
Normal & 13 & $6.02 \pm 3.91$ & 0.5 \\
LG & 2 & $8.86 \pm 7.78$ & 1.5 \\
FSH & 3 & $7.21 \pm 1.29$ & 0.7 \\
LG + FSH & 5 & $7.87 \pm 2.28$ & 1.0 \\
& & & \\
Tibialis anterior & & & \\
Normal & 11 & $7.87 \pm 1.30$ & 0.2 \\
LG & 22 & $8.38 \pm 3.45$ & 1.0 \\
FSH & 11 & $7.52 \pm 2.28$ & 1.5 \\
LG + FSH & 33 & $8.09 \pm 3.10$ & 1.2 \\
\hline
\end{tabular}

mal muscles. The maximal recorded length was about $15 \mathrm{~mm}$ in both muscles in the patient group. This is similar to the findings in normals.

In the dystrophic muscles the motor unit activity seemed to change more dramatically compared with normals, with abrupt changes in amplitude and duration of the unit. Within a unit, the appearance of the activity could vary considerably from being simple to highly complex and polyphasic and also from short to long duration. The units were significantly more often fragmented into groups which were separated by silent areas.
A muscle biopsy had been performed in 16 of the patients (although some years previous to this study in some patients). The LG patients had all clearly abnormal biopsies with increased fibre diameter variation, often fibre splitting, but no type grouping. There was no apparent decrease in number of fibres per unit area where this could be appreciated. There was an increased amount of connective tissue and fat. The FSH patients biopsies showed similar but often less pronounced changes. The early cases and the clinically less involved muscles showed only slight abnormalities with some variability of the fibre diameter. $^{3}$

\section{Discussion}

The fibre density was significantly increased in the brachial biceps and tibialis anterior muscles in the patients with muscular dystrophy. This increase has been discussed in a previous paper. ${ }^{3}$ The main reasons seem to be a locally increased number of muscle fibres due to processes such as fibre splitting, innervation of sequestered fibres, generation of new muscle fibres from satellite cells and ephaptic transmission between fibres. 
Macro EMG was used to study the total activity in the motor unit.9 Simulation studies ${ }^{12}$ indicate that there is a linear correlation between fibre size as well as number of fibres in the unit and the resulting Macro MUP amplitude and area. The $50 \%$ of the fibres most adjacent to the recording electrode contribute $70 \%$ of the Macro signal.

One might expect the Macro MUPs to be small in muscular dystrophies but this was not the case in the present study. Most Macro MUPs were normal or only slightly reduced in amplitude (although the difference from normal values was significant for the pooled material), even in severely weak muscles. This was in spite of other electrophysiological and morphological abnormalities such as an increase in FD and abnormal Macro MUP shape in 50\% of the recordings as detected by the pattern recognition technique, and also abnormal muscle biopsies indicating that the motor unit was involved by the disease.

May technical factors make the amplitudes erroneously high? The possibility of summation of activity from other units was tested in two ways. When recording the same motor unit during minimal and during forceful contraction (more than normally used in the main study) an amplitude increase could be obtained. At this moment the computer analysis usually indicated semisynchronous activity, and such recordings were not included in the material. When an increasing tremor was introduced on purpose, the semisynchronous activity of motor units was clearly revealed by the computer before any change in the Macro MUP occurred. In the actual patient recordings, no semisynchronous activity was accepted. A complete synchronicity between motor units cannot be detected with this technique but there is no evidence for such a phenomenon in muscular dystrophies. We therefore believe that the Macro MUPs reflect mainly undisturbed individual motor units.

Since technical factors cannot explain the relatively high Macro MUPs other mechanisms must be considered. The typical morphological findings in dystrophic muscles is increased fibre diameter variation and decreased mean fibre diameter ${ }^{13}$ In computer simulation the effect of pure atrophy on Macro MUP has been studied. ${ }^{12}$ With a decrease in average fibre diameter from $55 \mu \mathrm{m}$ to $43 \mu \mathrm{m}$ and normal or increased diameter variation the Macro MUP amplitude was reduced by up to $24 \%$. When the atrophy was accompanied by a corresponding general shrinkage of the motor unit territory the Macro MUP was only slightly reduced compared with values obtained with normal motor unit parameters. This is due to a reduced average distance between the electrode and the fibres. The model is in agreement with findings of slightly reduced Macro MUPs in disuse atrophy with a normal number but atrophic muscle fibres and reduced muscle volume (unpublished observation). In Scanning EMG there was no significant difference in motor unit cross section in muscular dystrophies, even in atrophic muscles, compared with normal. Thus there was no evidence of pronounced motor unit shrinkage. In summary the effect of atrophy on the Macro MUP may theoretically be counteracted by shrinkage and there are no indications that the amplitude will be increased above normal.

Another factor of importance for the size of the Macro MUP is the temporal dispersion of the single fibre action potentials within the motor unit. An increased synchronicity of action potentials should increase the Macro MUP, but such a situation is unlikely to occur. A desynchronization on the other hand of the action potentials in the motor unit in muscular dystrophies is more likely. This is supported by the observations of: increased variability of fibre diameter typically seen in muscular dystrophies, ${ }^{13}$ a greatly increased end-plate scatter in Duchenne dystrophy, ${ }^{14}$ an increased variability in propagation velocity among dystrophic muscle fibres $^{2}$ and an increased mean duration of spike components in SFEMG recordings. ${ }^{3}$ Increased time dispersion should lead to a reduction in MUP amplitude and to a lesser extent in area, according to the simulation studies. ${ }^{12}$ An increased area/amplitude ratio was actually found in the present recordings, particularly for low amplitude Macro MUPs. However the increased baseline noise contribute to the area parameter and the significance of the changed ratio is therefore uncertain.

A changed recruitment order or selective loss of motor units should influence the Macro EMG results. In normal subjects larger Macro MUPs are recruited with increasing muscle strength (unpublished data). Since the Macro measurements are usually obtained at a low degree of activation, a disturbed recruitment pattern could result in the sampling of larger motor units. There are no indications of an altered recruitment order in muscular dystrophies compared with normal. A selective loss of small motor units could also result in increased average amplitude of the Macro MUPs. There are no morphological findings indicating loss of motor neurons, ${ }^{15}$ although there are reports of altered motor neuron function seen as slowing of nerve conduction in the muscular dystrophies. ${ }^{16}$ Contradicting results have been reported. ${ }^{17}$ McComas found a decreased number of functional motor units but a normal mean motor unit amplitude in dystrophies. ${ }^{18}$ Others have not found reduced number of functional motor units. ${ }^{17} 19$

Altered volume conduction in muscular dys- 
trophies could be of importance for the Macro MUPs. The decline of the single fibre action potential over short distances (up to $455 \mu \mathrm{m}$ ) was the same in dystrophies as in normals. ${ }^{3}$ Altered volume conduction over longer distances may still be present. A morphological hallmark of muscular dystrophies is the increased amount of connective tissue and fat and an increasing separation of stroma and parenchyma ${ }^{20}$ It can be inferred that connective tissue and fat have higher resistance values than mus$\mathrm{cle}^{21}$ and volume conduction will probably decrease across bands of stroma. When a CNEMG recording is performed just outside the muscle fascia, it can be seen that the fibrous tissue causes low pass filtering of the EMG signal, and this is probably also true for intramuscular connective tissue. This is possibly the cause for the increased fragmentation of motor units seen in Scanning EMG. The Macro EMG mainly records low-frequency components which are not much affected by these changes in volume conduction. It is however likely that the relative importance of the closest fibres compared to the distant is more pronounced than in normal muscle.

The factors so far discussed do not seem to increase falsely the Macro MUP amplitudes in muscular dystrophies. Therefore the normal or only slightly reduced Macro MUPs may indicate that there is no major loss of electrical generators, that is, muscle fibres or fibre segments in the dystrophic motor units compared with normal muscle. The amplitude may be the resultant effect of fibre loss, atrophy, increased time dispersion counteracted by shrinkage, formation of new muscle fibres and ephaptic transmission between fibres.

The FD measurements indicates an increase in fibres per motor unit by a factor 2.4 in biceps and 1.9 in tibialis anterior when homogeneous fibre dispersion is assumed, calculated according to the description by Gath and Stålberg ${ }^{22}$ while the Macro amplitudes are decreased to 0.90 and 0.80 of the normal respectively. This discrepancy is considered to indicate a heterogeneous fibre distribution within the motor unit.

Scanning EMG showed no significant reduction in the diameter (cord) ${ }^{3}$ of the motor unit territory in these patients compared with normals. This is in agreement with findings of Buchthal et al in a study of the size of the motor unit territory with a multi electrode technique in muscular dystrophies. ${ }^{23}$ But the Scanning EMG topography was different in muscular dystrophies. The motor unit showed areas of activity, with abruptly changing configuration and amplitudes sometimes with silent portions. Fibre branching alone cannot give this picture. A variation in conduction velocity would give a large variation in latencies but no silent portions. The silent portions could either be due to loss of fibres in the motor unit or to fibrosis. The latter is not always the reason since activity from other motor units was sometimes recorded in the "silent areas" seen in some of the motor units.

The Scanning EMG thus seems to support the hypothesis of a clustering of muscle fibres within the dystrophic motor unit with local grouping mixed with silent areas. The heterogeneous fibre distribution also causes slightly more variation in MUP shape when recording from different areas within the same motor unit than seen in normal muscles.

These findings are in agreement with our earlier reported findings in single fibre EMG in muscular dystrophies, where a myogenic process such as muscle fibre branching is indicated by abnormally low jitter and a neurogenic process is seen as increased jitter and blockings. ${ }^{3}$ These processes could participate in the cycles of fibre degeneration and regeneration $^{20}$ that probably bring about the remodelling of the motor units.

How can the characteristic changes in CNEMG be explained if the Macro EMG indicates a near normal number of fibres and single fibre EMG indicates a local increase in fibre density? It seems that changed volume conduction properties may alter the CNEMG recording more than the SFEMG or Macro recordings. Distant fibres, for example, in neighbouring fascicles that normally contribute to the CNEMG MUP with spiky components may be sealed off. Therefore the main component of the MUP represents only the closest fibres. Owing to small grouping polyphasic MUPs will be seen with reduced contribution from distant fibres due to the filtering effects. The SFEMG recording has a small uptake area over which no volume conduction changes can be detected. ${ }^{3}$ Macro EMG on the other hand records low frequency components ${ }^{12}$ less influenced by fibrosis. Furthermore the electrode is passing physically through many fascicular boundaries reaching into different fascicles.

The discrepancy between normal Macro MUPs and reduced muscle strength needs some comments. This finding could either be due to fibre atrophy and general shrinkage which could give relatively normal Macro MUPs or be due to abnormal mechanical properties in the regenerating structures or a primary defect in contractile properties in myopathic fibres. This last possibility is supported in the patients by the presence of weakness, without atrophy and normal Macro MUP amplitudes. A hypothetical involvement of the muscle spindles in muscular dystrophies could affect muscle performance. This needs to be studied.

It was noted that the largest Macro MUPs were found in young patients with FSH. This could indi- 
cate an early compensatory hypertrophy in muscular dystrophies, detectable in Macro EMG. Such a mechanism is also reported in the literature. ${ }^{13}$ The dynamic changes in the dystrophic motor unit need longitudinal studies over time in the same patient.

This investigation was supported by the Swedish Medical Research Council, Grant 135.

\section{References}

' Stålberg E, Trontełj $\mathfrak{N}$, Janko M. Single Fibre EMG findings in muscular dystrophy. In: HausmanowaPetrusewicz I. and Jedrzejowska H, eds. Structure and Function of Normal and Diseased Muscle and Peripheral Nerve. Warsaw: Polish Medical Publisher, 1974;185-190.

${ }^{2}$ Stålberg E. Electrogenesis in human dystrophic muscle. In: Rowland L, ed. Pathogenesis of Human Muscular Dystrophies. Excerpta Medica 1977;404:570-87.

${ }^{3}$ Hilton-Brown P, Stålberg E. The Motor Unit in Muscular dystrophy, a Single Fibre EMG and Scanning EMG study. J Neurol Neurosurg Psychiatry 1983;46:981-995.

${ }^{4}$ Stålberg E, Trontelj JV. Single Fibre Electromyography. Woking, England: Mirvalle Press, 1979.

${ }^{5}$ Buchthal F. Diagnostic significance of the myopathic EMG. In: Rowland L, ed. Pathogenesis of Human Muscular Dystrophies. Amsterdam: Excerpta Medica 1977;404:205-18.

- Thiele B, Böhle A. Anzahl der Spike-Komponenten im Motor-Unit Potential. EEG EMG 1978;9:125-30.

${ }^{7}$ Stålberg E. Macro EMG, a new recording technique. $J$ Neurol Neurosurg Psychiatry 1980;43:475-82.

${ }^{8}$ Stålberg E, Antoni L. Electrophysiological cross section of the motor unit. J Neurol Neurosurg Psychiatry 1980;43:469-74.

${ }^{9}$ Stålberg E, Fawcett P. Macro EMG in healthy subjects of different ages. J Neurol Neurosurg Psychiatry 1982;45:870-78.

${ }^{10}$ Nandedkar S, Stâlberg E, Sanders D, Anne A, Kim Y. Use of Signal Representation to Identify Abnormal
Motor Unit Potentials in ALS. IEEE Trans Biomed Eng (in press).

"Stålberg E, Thiele B. Motor unit fibre density in the extensor digitorum communis muscle. J Neurol Neurosurg Psychiatry 1975;38:874-80.

12 Nandedkar S, Stålberg E. Simulation of Macro EMG motor unit potentials. Electroencephalogy Clin Neurophysiol (in press).

${ }^{13}$ Dubowitz V, Brook M. Muscle Biopsy: a Modern Approach. Philadelphia: WB Sanders, 1973.

${ }^{14}$ Coers C, Telerman-Toppet N. Morphological changes of motor units in Duchenne's muscular dystrophy. Arch Neurol 1977;34:396-402.

${ }^{15}$ Tomlinson BE, Walton JN, Irving D. Spinal cord limb motor neurons in muscular dystrophy. $J$ Neurol Sci 1974;22:305-27.

${ }^{16}$ Ballantyne JP, Hansen S. Neurogenic influence in muscular dystrophies. In: Rowland L, ed. Pathogenesis of Human Muscular Dystrophies. Amsterdam: Excerpta Medica, 1977;187-199.

${ }^{17}$ Panayiotopoulos CP, Scarpalezos S. Muscular dystrophies and motoneuron diseases. Neurology (Minneap) 1976;26:721-5.

${ }^{18}$ McComas AJ, Sica REP, Campbell MJ. Numbers and sizes of human motor units in health and disease. In: Desmedt JE ed. New developments in Electromyography and Clinical Neurophysiology, vol.l Basel: Karger 1973;55-63.

${ }^{19}$ Ballantyne JP, Hansen S. New method for the estimation of the number of motor units in a muscle. 2. Duchenne, limb-girdle and facioscapulohumeral, and myotonic muscular dystrophies. J Neurol Neurosurg Psychiatry 1974;37:1195-201.

${ }^{20}$ Schmalbruch $\mathrm{H}$. The muscular dystrophies. In: Mastaglia FL, Walton J, eds. Skeletal Muscle Pathology 1982;235-65.

2 Geddes LA, Baker LE. The specific resistance of biological material-A compendium of data for the biomedical engineer and physiologist. Med Biol Engng 1967;5:271-93.

${ }^{22}$ Gath I, Stålberg E. On the measurement of fibre density in human muscles. Electroencephalogy Clin Neurophysiol 1982;54:699-706.

${ }^{23}$ Buchthal F, Rosenfalck P, Ermino F. Motor Unit territory and fibre density in myopathies. $J$ Neurol 1960;10:398-408. 